\title{
Ann Arbor Clinical Stage III
}

National Cancer Institute

\section{Source}

National Cancer Institute. Ann Arbor Clinical Stage III. NCI Thesaurus. Code C125476.

A clinical stage that refers to lymphoma involving node regions on both sides of the diaphragm, or an extralymphatic organ or site plus nodes on both sides of the diaphragm (IIIe), or lymph nodes on both sides of the diaphragm plus spleen (IIIs). 